\title{
ACUTE GASTRIC DILATATION IN A 13 YEAR OLD CHILD. A CASE REPORT
}

\author{
Elīna Kalnakārkle*, Zane Ābola*, ** \\ * Riga Stradins University \\ ** Children's Clinical University Hospital, Latvia
}

\begin{abstract}
Summary
Acute gastric dilatation is a rare and life threatening disease. The usual symptoms of acute gastric dilatation can vary and be nonspecific, including acute abdominal pain, abdominal distension, nausea and vomiting. Many aetiologies of acute gastric dilatation are described. This article presents a case of a 13 year old girl who presented in our clinic with acute gastric dilatation after an orthopaedic operation. Clinical signs and a diagnostic pathway are presented in the report. In the early treatment of acute gastric dilatation, nasogastric tube insertion was used for gastric decompression. As a surgical approach jejunostomy insertion was used to ensure enteral feeding. Our case study shows that the optimal treatment for this pathology is mainly conservative.
\end{abstract}

Keywords: acute, gastric, dilatation, child

\section{AIM OF THE DEMONSTRATION}

The aim of the demonstration is to give an introduction to acute gastric dilatation in children and its nonspecific clinical signs and therapy options.

Case presentation: A 13 year old girl underwent an orthopaedic operation for juvenile idiopathic scoliosis. There were difficulties with intubation before operation. In the post-operative period she remained in the intensive care unit (ICU) for 4 days.

On the 5th post-operative day, a nasojejunal feeding tube (NJFT) was inserted to ensure enteral feeding. Enteral feeding had begun, but gastric retention appeared. After a few hours the patient had complaints about diffuse abdominal pain and abdominal distension. Sounds of bowel movement were absent. The enteral feeding through NJFT was cancelled. Plain abdominal $\mathrm{x}$-ray helped to visualize air-fluid levels and massive dilatation of the stomach (Fig. 1). A central venous catheter was inserted to ensure parenteral feeding. The patient was transferred to the surgical department with non-functional NJFT and parenteral feeding via central venous catheter, but despite total parenteral nutrition diffuse abdominal pain and abdominal distension was observed. A nasogastric tube was inserted for gastric decompression. The massive gastric fluid retention, which was approximately $500 \mathrm{ml}$ appeared, the next morning at approximately $1000 \mathrm{ml}$. An x-ray with contrast fluid was performed, contrast fluid was given through NJFT, and retention was detected (Fig. 2). Jejuno-gastro-oesophageal reflux was observed.

Surgical jejunostomy was created. The patient was transferred to the ICU where she remained on conservative treatment. Enteral feeding and parenteral feeding began. A few days after recovery in the ICU, the patient was transferred to the surgery department for further therapy. After creation of surgical jejunostomy retention through the nasogastric tube was still observed. There was gastric fluid retention every day of approximately $500-1500 \mathrm{ml}$.

\section{DISCUSSION}

Acute gastric dilatation (AGD) can be treatable if it is diagnosed early. But - if not recognized - mortality is as high as $80 \%(5,6)$. From published sources one may conclude that this pathology is rare in children. It is a multifactorial disease, so it is a challenge to prevent it. It is important to understand the clinical signs of the disease and react immediately. (1) Several aetiologies are mentioned in the literature to explain the pathogenesis including abdominal trauma, spinal surgery, eating disorders (anorexia nervosa and bulimia), abdominal vascular insufficiency, which has been associated with anaesthesia and spinal injuries. $(1,2,5)$ An $x$-ray can help recognise the anatomical cause of AGD, but the literature suggests that a CT scan of the abdomen is the most useful diagnostic investigation. $(5,6)$ Sometimes an endoscopy is performed to determine the condition of the gastric mucosa and to rule out any obstruction. (6) In this case, the AGD was diagnosed by the patient's nonspecific complaints, clinical signs and findings on plain $\mathrm{x}$-ray and $\mathrm{x}$-ray with contrast fluid. Nasogastric decompression usually reveals a massive volume of gastric fluid, as reported in our case. (5) In this case aerophagia can be the reason for AGD due to the relaxation of the upper oesophageal sphincter as a sequel to anaesthesia. (6) A genetic neurological disorder in our patient cannot be excluded, because from anamnesis is known that there were problems with preoperative intubation. As the literature and this case indicate, aggressive surgical treatment is not needed in AGD. The main role in treatment is gastric decompression by nasogastric tube, and jejunostomy to ensure enteral feeding. Surgical treatment is needed, if signs of peritonitis appear or if acute abdominal 
pathology cannot be ruled out. $(2,4)$ Operative intervention to relieve the cause of the obstruction and evaluate the gastric tissue is required, if the patient fails to improve. If AGD is left untreated, it can result in gastric necrosis and gastric perforation. (5) Distended stomach can compress aorta and inferior vena cava, which can result in limb ischemia. (7) The importance of recognising AGD lies in the fact that it can be potentially lethal. (3) It is not necessary to use aggressive surgical treatment for AGD. Mortality rate is not high, if AGD is recognized early and treatment with gastric decompression, parenteral and enteral jejunal feeding is started early.

\section{Conflict of interest: None}

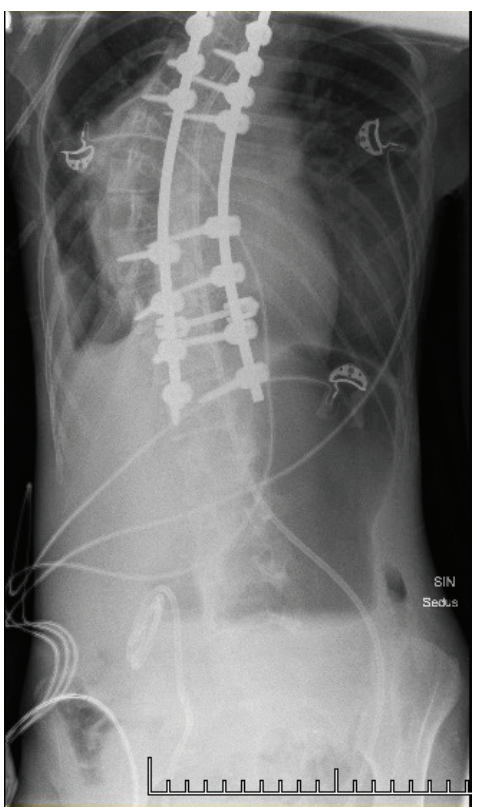

\section{REFERENCES:}

1. ToddSR, Marshall GT, Tyroch AH. Acute gastric dilatation revisited // The American Surgeon, 2000;709-710

2. D'yakonovax E, Bekin A,Poddubniy I, Isaev A, Nakovkin O., Krasavin A, Malashenko A. Acute gastric dilatation in children // Pediatric pharmacology, 2014;1 1.99. 10.15690/pf.vl li3.1018

3. Rastogi R, Rastogi V, Sachdeva IS, Bhargava S, Rastogi V. Acute gastric dilatation secondary to septicemia in newborn: A rare entity // African Journal of Pediatric Surgery, 2009;55-57

4. Qin H, Yao H, Zhang J. Gastric rupture caused by acute gastric distention in non-neonatal children: clinical analysis of 3 cases // Chinese Medical Journal, 2000;1147-1149

5. Moslim MA, Mittal J, Falk GA, Ustin JS, MorrisStiff G. Acute massive gastric dilatation causing ischaemic necrosis and perforation of the stomach // BMJ Case Reports, 2017; doi:10.1136/bcr-2016218513

6. Roy P, Mukherjee R, Parik M. An Unusual Cause of Abdominal Pain // Oman medical Journal, 2019;266-267

7. Kim AY, Jung SY, Eun LY. Life threatening acute gastric dilatation with aorta compression in a 3 year old child // Official Journal of The Japan Pediatric Society, 2015;1184-1186

\section{Address:}

Elīna Kalnakārkle, elina.kalnakarkle@rsu.edu.lv Asoc.Prof. Zane Ābola, zane.abola@rsu.lv

Fig. 1.

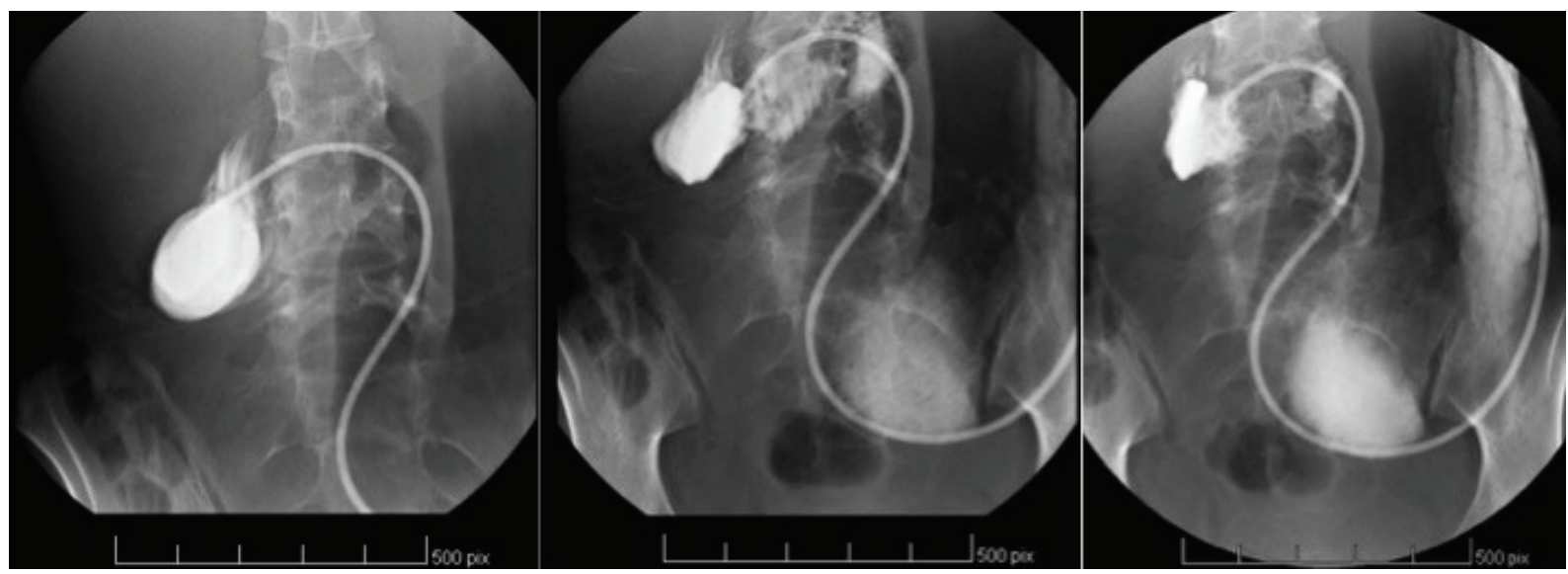

Fig. 2. 